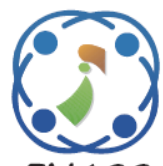

\title{
A Neural Network based Integer Frequency Offset Estimation and PSS Detection in 5G NR Systems
}

\author{
Vibha Patel ${ }^{1,2 *}$ \\ Krishna Warhade ${ }^{2}$ \\ ${ }^{1}$ Gujarat Technological University, Ahmedabad, India \\ ${ }^{2}$ School of Electronics and Communication Engineering, \\ Dr. Vishwanath Karad MIT World Peace University, Pune, India \\ * Corresponding author's Email: vibha.patel@mitwpu.edu.in
}

\begin{abstract}
With an increase in defined carrier frequency for the 5G new radio system, the need for synchronization also increases. The transceiver loses synchronization due to the occurrence of timing and carrier frequency offsets. Carrier frequency offsets often occur due to mismatch between transmitter and receiver oscillator frequency as well as the occurrence of doppler shifts due to transmitter/receiver movements. When frequency offsets exceed subcarrier spacing, integer frequency offset occurs that results in performance loss due to subcarrier indices shifts. Conventional approach i.e. maximum likelihood and sequential method is already employed to estimate integer frequency offset and to detect sector id. In this paper, the deep learning-based method is demonstrated to estimate the integer frequency offset and sector id detection. The neural network containing multiple convolution layers with activation layers is used to find the optimum received signal. Then, by calculating the number of cyclic shifts in the optimum received signal, the integer frequency offset is estimated. Using the corrected optimum received signal, the primary synchronization signal is also detected that gives sector id. This proposed estimator is tested for different profiles of tapped delay line models with different desired delay spread and compared with conventional methods i.e. maximum likelihood estimation method and sequential estimation method. Simulation results show that the proposed Neural Network based estimator outperforms in all delay profiles.
\end{abstract}

Keywords: 5G new radio, Integer frequency offset, Primary synchronization signal, OFDM, Neural network.

\section{Introduction}

The Fifth Generation New Radio (5G NR) system is a new interface technology developed by the $3 \mathrm{rd}$ Generation Partnership Project (3GPP), an extended version of the Long Term Evolution-Advance (LTEA) standard. It focuses mainly on three use cases, Ultra-Reliable Low Latency Communication (URLLC), Enhanced Mobile Broadband (eMBB), and Massive Machine Type Communications (mMTC) [1]. To support use cases, 3GPP modified the physical layer of 5G NR that supports large bandwidths, Multiple Inputs Multiple Outputs(MIMO), and Beamforming [1-3]. The operating bands of 5G NR are classified into two bands e.g. FR1- sub-6 GHz (below 6GHz) and FR2mmWave (above 24GHz) [4]. Orthogonal Frequency
Division Multiplexing (OFDM) is a key technique used in $3 \mathrm{G}$ and $4 \mathrm{G}$ Long Term Evolution (LTE) because it provides high data rate and spectral efficiency in a multipath environment. To support services defined in 5G, OFDM numerology is suitable for 5G NR physical layer [1].

However, OFDM faces major challenges i.e. Peak to Average Power Ratio (PAPR) and high sensitivity to timing and frequency offsets. In 5G, with an increase in defined carrier frequency, synchronization challenges also increase. InterCarrier Interference (ICI) is increased due to the large value of timing and frequency offsets. To make system ICI free, stable, and precise oscillators are required at the transmitter and receiver side $[5,6]$. In addition to that, Doppler shifts in received signal occur due to motion of transmitter or receiver results in performance degradation [7]. Hence, it becomes 
crucial to address synchronization issues by estimating and compensating timing offsets and carrier frequency offsets. Normalized carrier frequency offsets to subcarrier spacing are divided into two parts i.e. Fractional Frequency Offsets (FFOs) and Integer Frequency Offsets (IFOs). Due to fractional frequency offsets, orthogonality between subcarriers is destroyed, which leads to performance degradation. Integer frequency offsets leads to performance degradation due to the occurrence of cyclic shift in the frequency domain samples equal to integer value [8]. Timing offsets and Fractional Carrier Frequency Offsets (FCFO) are estimated and corrected before the FFT stage at the receiver side [9].

\subsection{Deep learning in wireless communication}

Wireless communication technologies are extensively developed to satisfy the applications and services in the wireless network. The $5 \mathrm{G}$ cellular network is designed to provide millisecond latency, thousand-fold capacity, and massive connectivity. Introduction of new technologies i.e. millimeter wave (mmWave), Ultra-Dense Network (UDN), and Multiple Inputs and Multiple Outputs (MIMO) make system design a more complex task. As stated in [11], 5G focuses on Device to Device communications (D2D) which increases the data traffic largely, making conventional communication systems difficult to operate. In addition to that, conventional communication system faces limitations to achieve a high data rate and to handle large data due to reasons i.e. Difficult channel modelling in complex scenarios, demand for effective and fast signal processing, and limited block-structure communication systems. Machine learning approach is already successful in the upper layers of the communication system. Deep learning used at the physical layer will lead to improved performance as compared to the conventional communication system.

In [12], the performance of deep learning based Non Orthogonal Multiple Access (NOMA), MIMO (Multiple Input Multiple Output), and millimeterwave (mmWave) is demonstrated. It is observed that deep learning methods perform superior to the conventional communication system.

In this paper, we propose a Neural Network $(\mathrm{NN})$ estimator for Integer Carrier Frequency Offsets estimation and Primary Synchronization Signal (PSS) detection. This proposed estimator is compared with conventional estimators i.e. Maximum Likelihood (ML) approach and sequential approach. The performance of proposed estimator is evaluated for different delay profiles of Tapped Delay Line
(TDL) channel model with various delay profiles. The main features of proposed estimator are:

1) The proposed $\mathrm{NN}$ estimator consisting Convolution Neural Network (CNN) layers and regression layer is trained and tested for various TDL channel models.

2) The received signals perturbed from IFOs and channel impairments are given to an input layer of proposed $\mathrm{NN}$ estimator. This estimator will find optimum received signal and will calculate number of cyclic shifts in the signal in order to estimate IFO and to detect sector ID.

3) This proposed $\mathrm{NN}$ estimator gives promising performance in all TDL delay models as compared to conventional estimation schemes and also provides wide IFO acquisition range of $[-3,3]$. To consider various $5 \mathrm{G}$ evaluation scenarios shown in [22], this TDL delay models are scaled to various delay spreads while training and testing.

Considering promising future of deep learning based wireless communication, this proposed $\mathrm{NN}$ estimator could be an encouraging solution to synchronization problem.

Deep learning approach in wireless communication is gaining popularity due to its ability to handle large amount of data and faster data processing. However, synchronization still remains critical issue in deep learning based wireless communication system also. In general, synchronization is achieved by estimating and compensating CFOs and timing offsets at appropriate stage at receiver side. Synchronization issue is addressed in conventional communication system through various methods i.e. autocorrelation, cross correlation and ML estimation. These methods are discussed in detail in section 2. Recently, the deep learning approach is employed to estimate CFOs which is discussed in section 2. Section 3 described $5 \mathrm{G}$ system model in detail including structure of synchronization signal, cell search procedure and Cyclic Prefix (CP) OFDM signal model. The proposed NN estimator is illustrated in details in section 3. In section 4, simulation results of proposed $\mathrm{NN}$ estimator are shown and compared with existing conventional estimation methods. Performance of proposed estimator is shown in terms of probability of failure in estimation of IFO and in detection of Sector ID. Also, probability of failure over estimation range is calculated and plotted, which measures 
performance of proposed estimator over normalized IFO in different TDL delay profiles.

\section{Related work}

\subsection{Conventional integer frequency offsets (IFOs) estimation methods}

In LTE, many methods are investigated for IFO estimation using PSS. In [13], the ML method is used to estimate IFO and sector ID jointly. This method is proved to be robust in multipath environments and to residual timing errors due to Approximated Minimum Mean Square Criterion (AMMSE) reduced rank presentation of Channel Frequency Response (CFR). In [14], IFO and sector ID are estimated sequentially. Proposed method achieves better accuracy at lower computational complexity as compared to conventional Maximum Likelihood (ML) methods. Low complexity joint estimation of IFO and Cell ID (CID) algorithm is proposed in [15]. Time-domain equalization with Maximum Likelihood estimation method is used to mitigate ICI in OFDM. In this paper, the authors achieved superior performance of TDE method over the conventional method of frequency domain equalization at low complexity. [9]

In [10], for 5G systems, autocorrelation and cross correlation based methods are demonstrated for FFOs estimation. When Carrier Frequency Offsets (CFOs) exceed subcarrier spacing then an autocorrelation based algorithm is used to estimate FFOs and IFOs, which can be estimated by calculating shifts of the received synchronization signal. Authors in [16] have proposed the detection of IFO and Sector ID sequentially without prior knowledge of PSS for 5G NR. This scheme provides low complexity at the cost of slight performance degradation. It uses fact that three PSS sequences generated for 3 Sector IDs are cyclically shifted versions of each other.

This existing conventional approach i.e. ML and sequential estimation for $5 \mathrm{G}$ NR system model is designed for conventional limited block structure communications. These estimation methods work on predefined system models and solve estimation problem by mathematical process [20]. The estimation accuracy degrades with change in system model, that results into less compatibility.

\subsection{Deep learning based carrier frequency offsets (CFOs) estimation methods}

In [17], CFO of a complex sinusoid is estimated using Deep Learning (DL) architectures. The achieved estimation range of CFO is $[0.2,0.25]$.
Deep Neural Network (DNN) based CFO estimation and packet detection schemes have been investigated and demonstrated for the emerging IEEE 802.11ah standard in [18]. In this paper, the Convolution Neural Network (CNN) and Recurrent Neural Network (RNN) architectures are used to train the model. And superior performance of DNN methods is demonstrated to conventional methods. This method estimates FFOs in the range [0.5,0.5]. In [19], authors have proposed a synchronization method based on Extreme Learning Machine (ELM) to estimate residual symbol timing offset and residual carrier frequency offset, which exhibits superior performance with existing traditional methods. The performance of the scheme is evaluated under Additive White Gaussian Noise (AWGN) channels and frequency selective fading channels.

The NN based coarse CFO estimator is proposed for multiple inputs and multiple output (MIMO). In this paper, the estimation problem is transformed into a classification problem and a training dataset is generated for normalized CFO range $(-0.5,0.5]$, which is fractional frequency offsets (FFOs). Furthermore, the model is trained for the AWGN channel, slow fading channel, and multipath channel for the different number of antennas [20]. These all estimation schemes are designed to estimate FFOs for different system models to be used in different applications.

However, to the best of the author's attention, in the 5G NR system, deep learning based IFO estimation is not explored extensively. In this paper, a Neural Network based estimator is proposed to estimate IFO and to detect sector ID (SID) for the $5 \mathrm{G}$ NR system. The proposed method is simulated for different delay profiles of the Tapped Delay Line (TDL) models scaled to desired delay spread. Here, the performance of the system is measured in terms of the probability of failure. The neural network is trained for IFO range [-3,3] and different delay profiles of TDL channel model. An infinite signal to noise ratio (SNR) is considered to avoid noise disturbance in the training process.

\section{System model}

\subsection{Synchronization signal}

As in LTE, User equipments (UEs) of $5 \mathrm{G}$ NR system also uses Primary Synchronization Signal (PSS) and Secondary Synchronization Signal (SSS) to detect frame boundary, sector identity (SID), and cell identity (CID) [1]. The structure of the Synchronization Signal Block (SSB) is shown in 


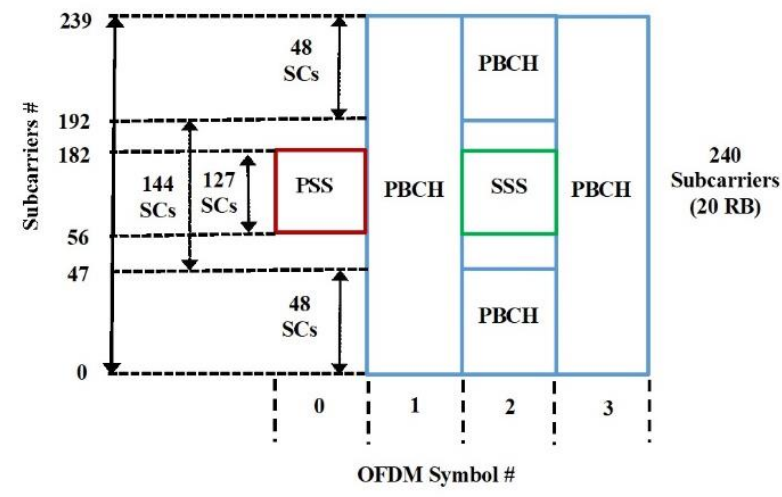

Figure. 1 Synchronization signal block (SSB) for resource allocation

Fig. 1. PSS is transmitted on the $0^{\text {th }}$ OFDM symbol followed by $\mathrm{PBCH}$, SSS is transmitted on $2^{\text {nd }}, 3^{\text {rd }}, 4^{\text {th }}$ OFDM symbols respectively. The SSB consists of 240 contiguous subcarriers (20 resource blocks), from which PSS symbols are transmitted on 144 subcarriers.

In $5 \mathrm{G}$, each cell has a unique cell identity (CID) number from 1008 cell IDs. These cells are arranged into 336 different groups represented as Group identity (GID). Each group is identified by three different sectors which are denoted by Sector identity (SID). The gNodeB transmits PSS and SSS periodically, which are used by the UEs to detect frame boundary and sector ID, synchronize, and identify the network.

PSS contains the SID index i.e. $u \in\{0,1,2\}$ and SSS contains the GID index i.e. $v \in\{0,1,2, \ldots, 335\}$.

The serving CID is given by

$$
N_{I D}=3 u+v
$$

Where $\mathrm{u}$ is integer valued SID and $u \epsilon\{0,1,2\}$, $\mathrm{v}$ is integer valued GID and $v \epsilon\{0,1,2 \ldots, 335\}$.

Similar to 4G networks, for 5G NR, PSS helps UEs to detect sector ID e.g. u, and to get radio frame boundary. NR PSS signal is assigned on the first symbol of each SSB and consists of one of three 127symbols $\mathrm{m}$-sequences. The three possible $\mathrm{m}$ sequences are defined as below [1]

$$
d_{P S S}(n)=1-2 x(m)
$$

Where,

$$
\begin{aligned}
& m=[n+43 u] \bmod 127, \quad 0 \leq n<127 \\
& x(i+7)=[x(i+4)+x(i)] \bmod 2
\end{aligned}
$$

and, $[x(6) x(5) x(4) x(3) x(2) x(1) x(0)]=$

[1 111100110$]$

These PSS symbols are transmitted on 56 to 182 subcarriers out of 240 subcarriers.

The 5G NR SSS is used to detect cell ID (CID) and has 336 combinations defined as below [1]

$$
\begin{aligned}
d_{S S S}(n)= & {\left[1-2 x_{0}\left(\left[n+m_{0}\right] \bmod 127\right)\right] \times[1-} \\
& \left.2 x_{1}\left(\left[n+m_{1}\right] \bmod 127\right)\right]
\end{aligned}
$$

Where,

$$
\begin{aligned}
& m_{0}=15[v / 112]+5 u \\
& m_{1}=v \bmod 112, \quad 0 \leq n \leq 127 \\
& x_{0}(i+7)=\left[x_{0}(i+4)+x_{0}(i)\right] \bmod 2 \\
& x_{1}(i+7)=\left[x_{1}(i+4)+x_{1}(i)\right] \bmod 2 \\
& {\left[x_{0}(6) x_{0}(5) x_{0}(4) x_{0}(3) x_{0}(2) x_{0}(1) x_{0}(0)\right]=} \\
& {\left[\begin{array}{lllllll}
0 & 0 & 0 & 0 & 0 & 0 & 1
\end{array}\right]}
\end{aligned}
$$

and,

$\left[x_{1}(6) x_{1}(5) x_{1}(4) x_{1}(3) x_{1}(2) x_{1}(1) x_{1}(0)\right]=$

$\left[\begin{array}{lllllll}0 & 0 & 0 & 0 & 0 & 0 & 1\end{array}\right]$

\subsection{Cell search procedure}

The 5G NR synchronization and cell search procedure are summarized in Fig. 2. First, timing, fractional frequency offsets, and frame timings are estimated and corrected in the time domain using autocorrelation and cross-correlation methods. Then, received corrected time-domain samples are converted into the frequency domain using FFT. After this, IFO is estimated by evaluating the frequency domain shifts in the received signal. After IFO correction, Sector ID $u$ is detected by crosscorrelating corrected received signal with 3 different $\mathrm{m}$ sequences, which represents sequence for sector ID 0,1 and 2. After PSS detection, cell id group $v$ (GID) is detected using SSS and SID. Hence, serving cell id

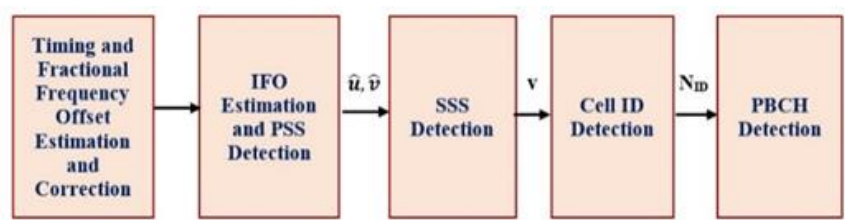

Figure. 2 Synchronization and cell search procedure 
can be calculated using Eq. (1). Here, the focus is on estimation of IFO and PSS detection using a neural network.

\subsection{Signal model}

The Cyclic Prefix Orthogonal Frequency Division Multiplexing (CP-OFDM) system is considered that consists of $N$ subcarriers with separation $\Delta f$ in the frequency domain. OFDM symbol at the transmitter is generated by performing $N$ point inverse fast Fourier transforms (IFFT) on modulated complex data. A cyclic prefix (CP) of length $N_{c p}$ is added OFDM symbol as a prefix to avoid Inter Symbol Interference (ISI). Usually, the length of $\mathrm{CP}$ is preferred greater than the channel delay spread. In 5G, normal and extended $\mathrm{CP}$ configurations are supported [1]. This leads to an increased time duration of one OFDM symbol, which results in total time duration $N_{t}=N_{c p}+N$. The transmitted OFDM symbol $s_{m}(n)$ for the $m^{\text {th }}$ period is given by

$$
s_{m}(n)=\sum_{k=0}^{N-1} S_{m}(k) e^{\frac{j 2 \pi k n}{N}}
$$

Where $n=-N_{c p},-N_{c p}+1, \ldots \ldots, N-1$ discrete-time index $S_{m}(k)$ is a complex signal transmitted on $\mathrm{k}^{\text {th }}$ subcarrier

$k=$ subcarrier index

The transmitted signal is distorted by a multipath channel which is represented by discrete-time impulse response $h_{m}(n)$. Then, complex Additive White Gaussian Noise (AWGN) is added to the distorted transmitted signal. The received signal is degraded due to $\mathrm{CFO}$, which is generated due to a mismatch between transmitter and receiver local oscillator and doppler effect when transmitter/ receiver is moving. At the receiver side, first, timing offset and FFOs are estimated and corrected. After FFT, IFO and sector id are estimated jointly or sequentially. Here, it is assumed that timing offsets and FFOs are estimated and corrected [21]. The received signal in the time domain for the $m^{\text {th }}$ period is given by

$$
\begin{gathered}
y_{m}(n)=e^{\frac{j 2 \pi(n-\vartheta)}{N}} S_{m}(n) * h_{m}(n)+w_{m}(n) \\
n=-N_{C P},-N_{C P}+1, \ldots \ldots, N-1 .
\end{gathered}
$$

Where $v$ is normalized IFO to subcarrier spacing, $h_{m}(n)$ is channel impulse response, $*$ is convolution operator and $w_{m}(n)$ is sampled zero mean white Gaussian noise with variance $\sigma_{w}^{2}$.

Once CP is removed, the time-domain signal is converted into a frequency-domain signal by performing FFT on the received signal. The signal in the frequency domain is given by

$$
\begin{gathered}
Y_{m}(k)=H_{m}(k-\vartheta) S_{m}(k-\vartheta) e^{\frac{j 2 \pi(k-\vartheta)}{N}}+W_{m}(k), \\
|\vartheta| \leq G \\
k=1,2,3, \ldots, N-1 \text { subcarriers }
\end{gathered}
$$

Where $H_{m}(k)$ is channel frequency response over $\mathrm{k}^{\text {th }}$ subcarrier with variance $\sigma_{H}^{2}, W_{m}(k)$ is complex zero-mean additive white Gaussian noise with variance $\sigma_{W}^{2}$ and $v$ is the normalized IFO to subcarrier spacing and $G$ is the maximum value of IFO.

\subsection{Conventional estimation methods}

Conventional method to estimate IFO and sector ID is presented in [13] and [16] for LTE and 5G system model. In [13], Maximum Likelihood estimation method is employed to estimate IFO and to detect PSS. These two parameters are estimated jointly. In this, the concentrated likelihood function is maximized for different values of IFO and sector ID. This concentrated likelihood function is defined by

$\hat{\varphi}_{M L}=\arg _{\varphi} \max \{f(\hat{\varphi})\}$

This likelihood function $f(\hat{\varphi})$ is defined by Eq. (15)

$f(\hat{\varphi})=\frac{1}{63\left\|Y_{m}\right\|^{2}}\left|\sum_{k=-31}^{31} Z_{m}(\hat{u}, \hat{v} ; k)\right|$

Where $Z_{m}(\hat{u}, \hat{v} ; k)=Y_{m}(k+\hat{v}) d_{P S S}{ }^{*}(k)$

Estimated values will be the ones for which likelihood function given in Eq. (15) will be maximized.

In [16], sequential approach is used to estimate IFO and sector ID. In this, cross correlation is performed between received signal and summed PSS sequences. This function is defined as

$$
\emptyset(m)=\sum_{k=57}^{182} Y_{m}(k+m) \bar{D}^{*}(k)
$$

Where $\bar{D}(k)=\sum_{w=0}^{2} D_{w}(k)$ is summed NR PSS, $\mathrm{m}=\{-3,-2, \ldots, 2,3\}$ trial values of IFO and $D_{w}(k)(w=0,1,2)$ represents three $\mathrm{m}$ sequences. They proposed method based on the fact that three $\mathrm{m}$ 
sequences are cyclically shifted versions of each other and therefore cross correlation between any pair of $\mathrm{m}$ sequences is nearly zero. This objective function is maximized for different hypothesized values of IFO. The estimated IFO is the one for which objective function is maximized. The estimated IFO is given by

$$
\hat{v}=\arg _{m} \max \Re\{\varnothing(m)\}
$$

Where $\Re$ indicates real part of the quantity. After IFO estimation, Sector ID is sequentially detected using

$$
\widehat{u}=\arg _{w} \max \mathfrak{R}\left\{\sum_{k=57}^{182} Y_{m}(k+\hat{v}) D_{w}^{*}(k)\right\}
$$

Where $w \in\{0,1,2\}$ is trial values of Sector ID.

As discussed here, these conventional estimation methods are based on predefined system model. Its performance degrades with change in system model that results into low compatibility. To provide high compatibility, deep learning approach is proposed here.

\subsection{Proposed neural network (NN) based IFO estimation}

Here, estimation problem is converted into regression problem. IFO and sector ID are estimated and detected using a neural network containing Convolution Neural Network (CNN) layers and regression layer as a final layer. The typical $\mathrm{CNN}$ is shown in Fig. 3. The CNN successfully captures temporal and spatial correlation in the input signal. Here, 2-D convolution layer is applied on received input signal. This proposed NN estimator consists of input layer, hidden layers and output layer. The regression layer as an output layer calculates half squared Mean Square Error (MSE). This proposed neural network is trained for several epochs to minimize MSE. Due to this, proposed NN estimator gives optimum performance when the impaired signal from channel nonlinearities and IFO is given to this trained network.

This proposed NN estimator takes received signal, learns estimation rule, and gives the best IFO candidate near to true IFO. To train the model, training datasets are generated for different values of IFO and different TDL channel delay profiles with various desired delay spreads [22]. The training datasets are divided into training data and validation data to avoid overfitting. First, the model is trained using training data and then examines the performance using validation data. The loss function is minimizing during the next epochs. After the

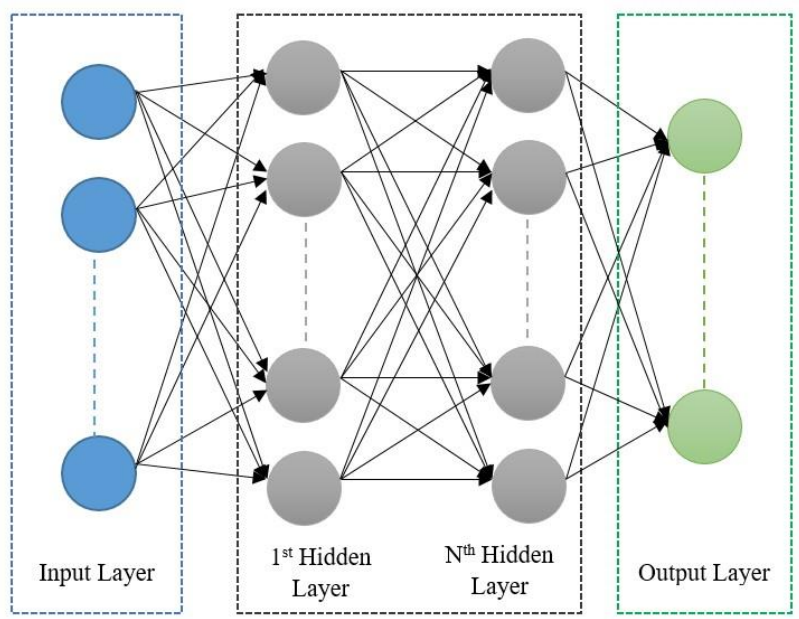

Figure. 3 Typical structure of convolution neural network

model is trained, test data set is given to trained NN estimator to find optimal IFO nearest to true IFO and sector ID.

CNN based IFO estimator accepts real and complex valued received signals separately. The architecture of NN based IFO estimator is shown in Fig. 4. The received signal is separated into real and imaginary dataset. In the next convolution layer, the column vector of 432 symbols is processed by 250 9x9 filters of convolution layers with a Rectified Linear Unit (ReLU) activation function to generate 250 outputs. Subsequently, six convolution layers of $5 \times 5$ filters

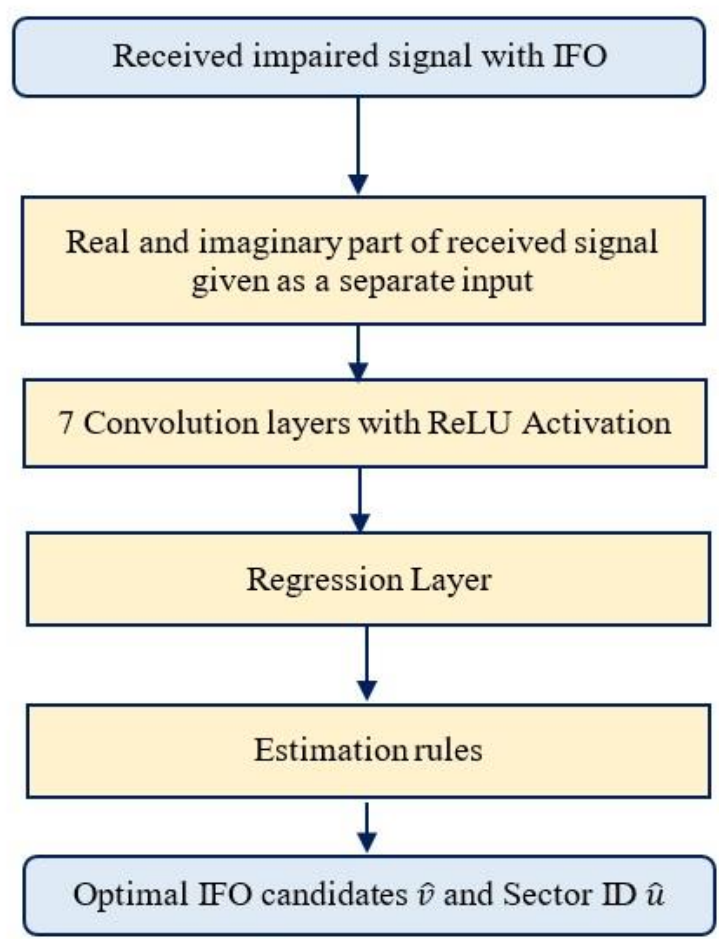

Figure. 4 NN based IFO estimator architecture 
are used followed by ReLU activation. At the output layer, an estimated real and imaginary part of the received signal is obtained that is nearer to true values of the real and imaginary part of the perturbed signal with IFO.

After this, estimated IFO can be calculated by finding the number of cyclic shifts in the estimated received signal by NN. The estimated received signal is corrected by estimated IFO in order to detect sector ID. The sector ID is detected by correlating corrected received sequence and three $\mathrm{m}$-sequences.

\section{Results and discussion}

For simulation, a $40 \mathrm{MHz} 5 \mathrm{G}$ NR system is considered with the number of subcarriers $\mathrm{N}=512$, symbol duration $\mathrm{T}_{\mathrm{s}}=0.016 \mu \mathrm{s}, \Delta \mathrm{f}=30 \mathrm{KHz}$ which is operating at $6 \mathrm{GHz}$ [16]. As per normal mode, a cyclic prefix of 44 symbols length is added. PSS of 127 symbols is transmitted on 56 to 182 subcarriers out of 240 subcarriers. For $5 \mathrm{G}$ channel model, the tapped delay line (TDL) model is considered with different delay profiles. These delay profiles are classified into Non-Line of Sight (NLOS) i.e. TDLA, TDL-B, TDL-C, and Line of sight (LOS) i.e. TDLD, TDL-E models. Power delay profiles of these channel models are described in [22]. Here, normalized IFO value is considered from -3 to 3 , by considering the stability of commercial oscillator of $\pm 20 \mathrm{ppm}$ for mobile applications [16]. Here, the performance of the estimation of IFO and sector id is separately shown. The performance metric is the probability of failure that is expressed as $\mathrm{P}_{\mathrm{fv}}=$ $\operatorname{Prob}\{(\hat{v}) \neq(v)\}$ for IFO and $\mathrm{P}_{\mathrm{fu}}=\operatorname{Prob}\{(\hat{u}) \neq(u)\}$ for Sector ID. The simulation parameters are shown in Table 1.

Here, the training dataset is generated across normalized IFO range $[-3,3]$ with resolutions of 1 and for 5 channel models as discussed earlier. There are 127 symbols in the PSS signal on 56 to 182 subcarriers. In training process, to avoid disturbance from noise, SNR is set to an infinite value. The proposed $\mathrm{NN}$ is trained for random value of IFO selected from the interval $[-3,3]$, and for randomly selected delay profiles. These generated delay profiles can be scaled to desired delay spread. We considered here five cases of delay spread very short delay spread (10ns), short delay spread (30ns), nominal delay spread (100ns), long delay spread (300ns), and very long delay spread (1000ns) while training the neural network.

In this way, 1,00,000 frames for training datasets are generated and these frames are perturbed from random IFO and random TDL profile with desired
Table 1. Simulation parameters

\begin{tabular}{|c|c|}
\hline Parameter & Value \\
\hline Carrier frequency & $40 \mathrm{MHz}$ \\
\hline Symbol duration $\mathrm{T}_{\mathrm{s}}$ & $0.016 \mu \mathrm{s}$ \\
\hline Subcarrier spacing & $30 \mathrm{KHz}$ \\
\hline Guard Interval $\mathrm{N}_{\mathrm{g}}$ & 44 \\
\hline FFT size, $\mathrm{N}_{\mathrm{FFT}}$ & 512 \\
\hline Channel Model & Tapped Delay Line (TDL) \\
\hline TDL delay profiles & $\begin{array}{r}\text { TDL-A, TDL-B, TDL- } \\
\text { C,TDL-D, TDL-E }\end{array}$ \\
\hline
\end{tabular}

scaled delay spread. Frequency selective fading environment is selected for training as well as testing purpose.

The batch size is set to 1000 in the training of the proposed NN estimator. Test data is given to the proposed NN estimator and tested for SNR value from $-6 \mathrm{~dB}$ to $15 \mathrm{~dB}$ and for different delay profiles. While testing, NLOS and LOS delay profiles are scaled with various delay spreads is selected to validate results in different scenarios. These simulation results are compared with the traditional ML estimator presented in [13] and sequential estimator presented in [16].

In Fig. 5, performance of the proposed NN estimator, ML method, and sequential method is shown for NLOS delay profiles TDL-A, TDL-B, and TDL-C. It is seen that the proposed NN estimator outperforms in all delay profiles as compared to ML and sequential methods. The proposed NN estimator achieves less probability of failure in less than $11 \mathrm{~dB}$ region for NLOS delay profiles. At failure probability of " $10^{-2}$ ", proposed $\mathrm{NN}$ estimator realizes $4 \mathrm{~dB}, 7 \mathrm{~dB}$ and $9 \mathrm{~dB}$ SNR in TDL-B, TDL-A and TDL-C delay profiles respectively. However, ML and sequential methods fails to achieve same failure probability.

Fig. 6 shows the performance of the proposed NN based IFO estimator and conventional methods for LOS delay profiles TDL-D and TDL-E. The proposed scheme again performs well and exceptionally well in all delay profiles concerning ML and sequential estimation respectively. The proposed scheme has an SNR improvement of $5 \mathrm{~dB}$ to ML estimation. For the negative SNR region, the NN estimator also performs well. Proposed estimator achieves " $10^{-2}$ " failure probability at SNR $3 \mathrm{~dB}$ and $4 \mathrm{~dB}$ in TDL-D and TDL-E delay profiles respectively. The same can be achieved at $6 \mathrm{~dB}$ and $7 \mathrm{~dB}$ SNR in ML method. However, sequential method fails to achieve same probability of failure.

In Fig. 7 and Fig. 8, the performance of the proposed NN based IFO estimator to ML and sequential method for sector ID estimation in presence of Non LOS and LOS delay profiles 


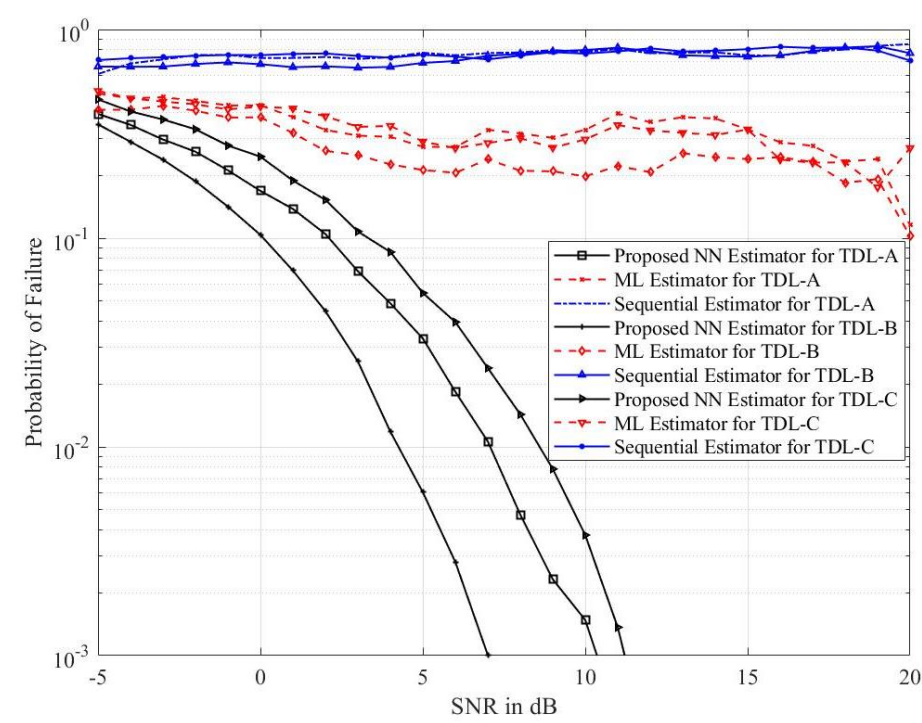

Figure. $5 \mathrm{P}_{\mathrm{fv}}$ vs. SNR of IFO estimation for NLOS delay profiles

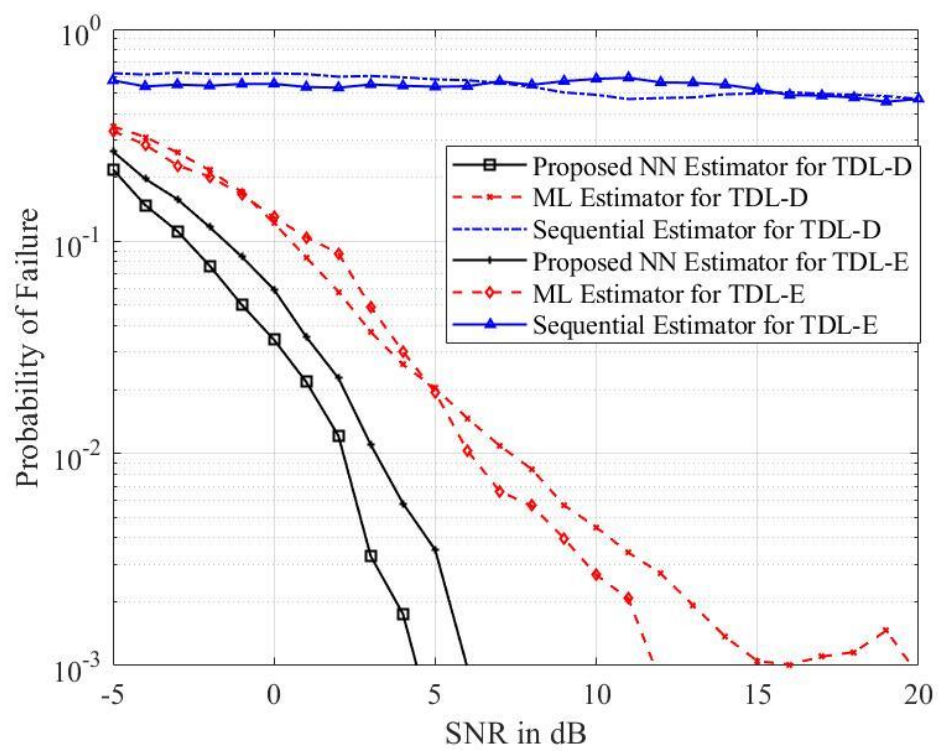

Figure. $6 \mathrm{P}_{\mathrm{fv}}$ vs. SNR of IFO estimation for LOS delay profiles

respectively. In Fig. 7, for negative and very low SNR region, Proposed NN and ML estimator performs same but proposed NN estimator improves in high SNR region. To achieve $P_{f u}$ of $10^{-2}$, proposed NN estimator requires $2 \mathrm{~dB}, 4 \mathrm{~dB}$, and $6 \mathrm{~dB}$ SNR for TDL-B, TDL-C, and TDL-A respectively. However, ML and sequential methods do not achieve the same even for high SNR region. In Fig. 8, it can be shown that the proposed NN estimator has SNR improvement in TDL-D profile as compared to ML and sequential estimation. The proposed $\mathrm{NN}$ estimator shows SNR improvement of $8 \mathrm{~dB}$ at $10^{-2}$ for TDL-E profile with respect to ML estimation. But, sequential method does not achieve the same target. It is observed in both figures that the NN based IFO estimator performs well in Non LOS and LOS delay profiles.

The performance of the proposed estimator is also shown concerning the IFO range. This performance is shown in terms of the probability of failure. Fig. 9 and Fig. 10 show the performance of the proposed $\mathrm{NN}$ based IFO estimator to sequential and ML estimator for NLOS and LOS delay profiles respectively. The NN estimator is trained for IFO range -3 to 3 , so it exhibits a low probability of failure in the specified range. when IFO exceeds the specified range, failure probability is increased.

In Fig. 9, NN based IFO estimator gives the almost same performance as the ML method but improved from the sequential estimator for delay 


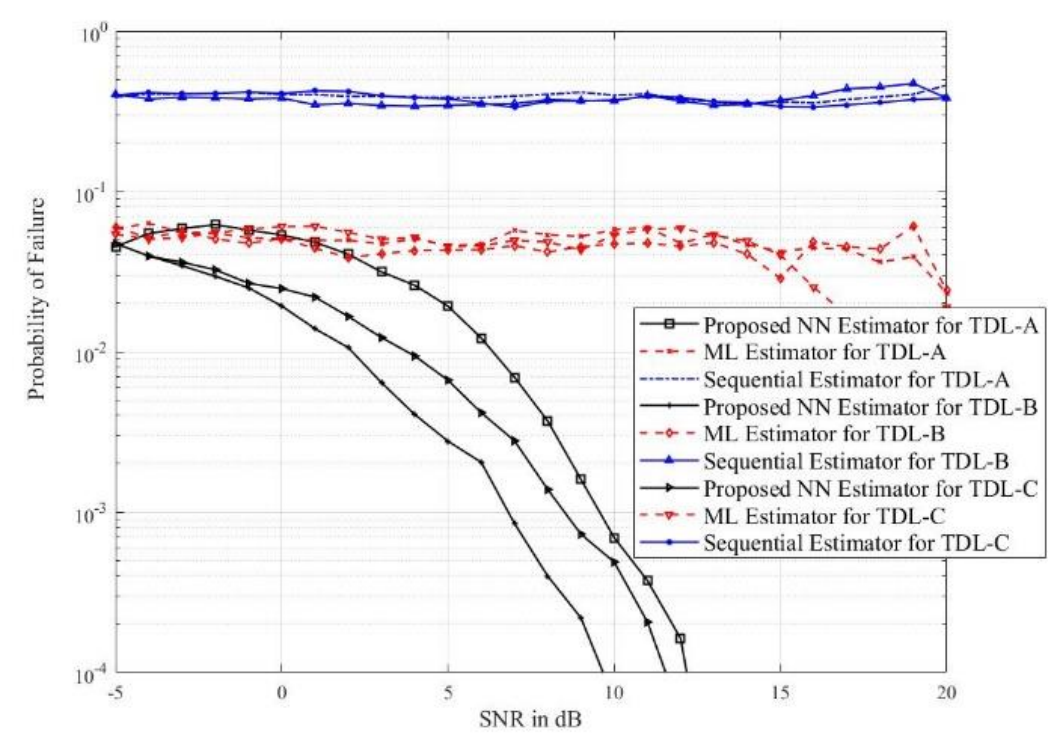

Figure. $7 \mathrm{P}_{\mathrm{fu}}$ Vs. SNR of Sector ID estimation for Non-LOS delay profiles

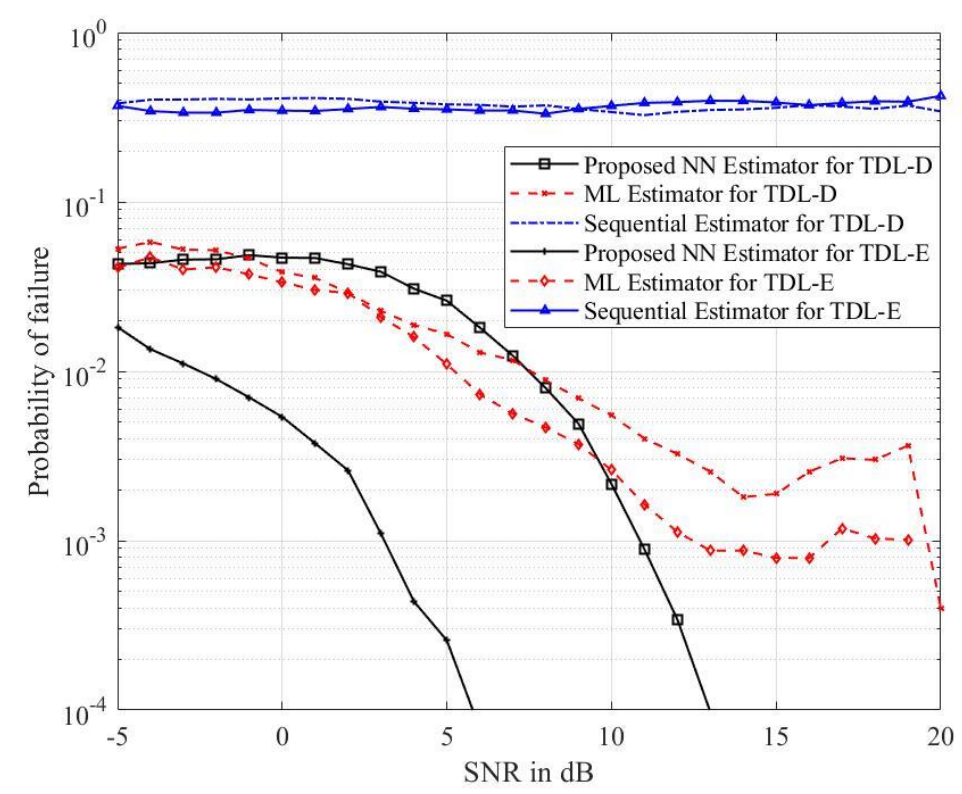

Figure. $8 \mathrm{P}_{\mathrm{fu}}$ vs. SNR of Sector ID estimation for LOS delay profiles

profiles TDL-A, TDL-B and TDL-C. In Fig. 10, the proposed $\mathrm{NN}$ estimator performs well as compared to the conventional methods for the specified IFO range. In all simulation results, it can be observed that the proposed NN estimation method works efficiently and gives less failure probability in all channel delay profiles. This occurs due to a proposed neural network is trained with different channel delay profiles and for different IFO values from -3 to 3 . And the trained neural network will find optimal received sequence when test data is given. These all delay profiles are scaled to appropriate delay spread and the proposed estimation method tested for the same.

\section{Conclusion}

In this paper, we propose $\mathrm{NN}$ based IFO estimation and PSS detection for the 5G NR system. The deep learning approach is employed over conventional methods to estimate IFO and to detect PSS. This proposed estimator is tested for different delay profiles of TDL channel and compared with conventional method ML and sequential estimation. These delay profiles are scaled with various desired 


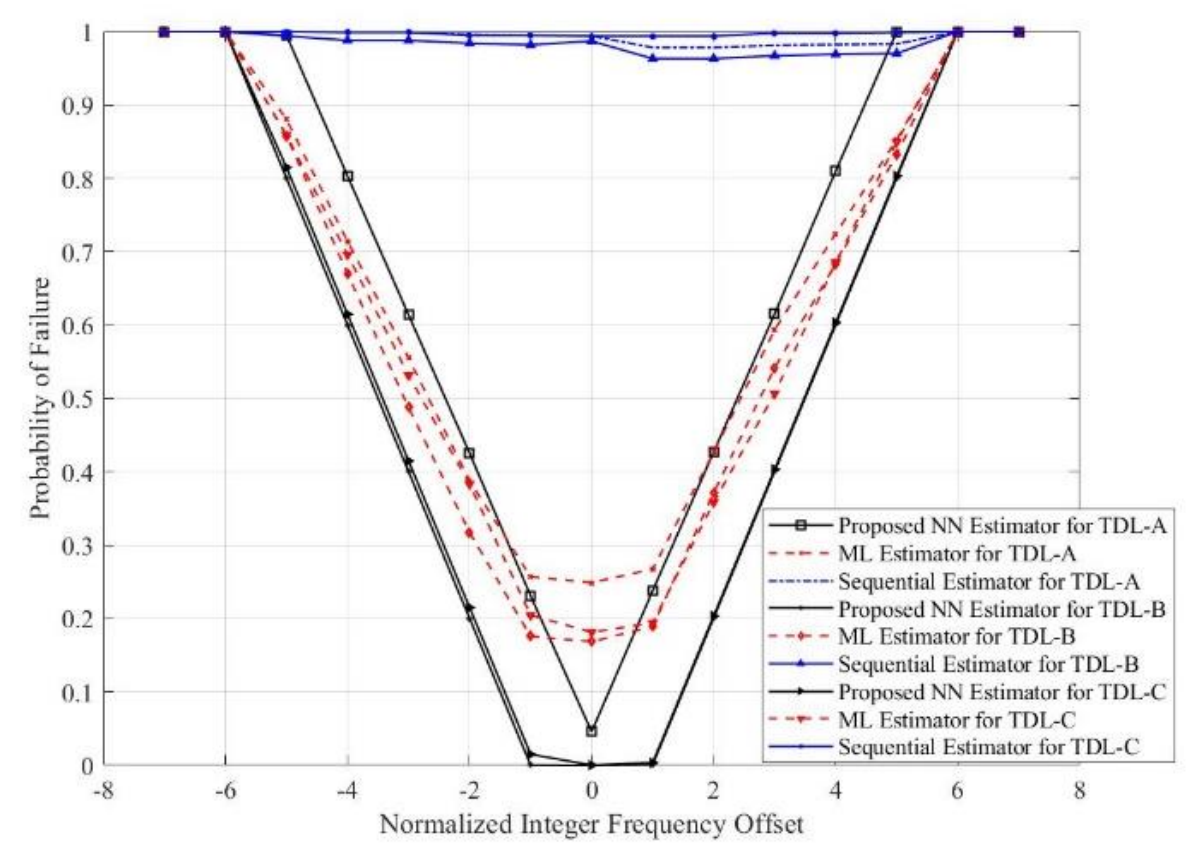

Figure. $9 \mathrm{P}_{\mathrm{f}}$ vs. Normalized IFO for NLOS delay profiles

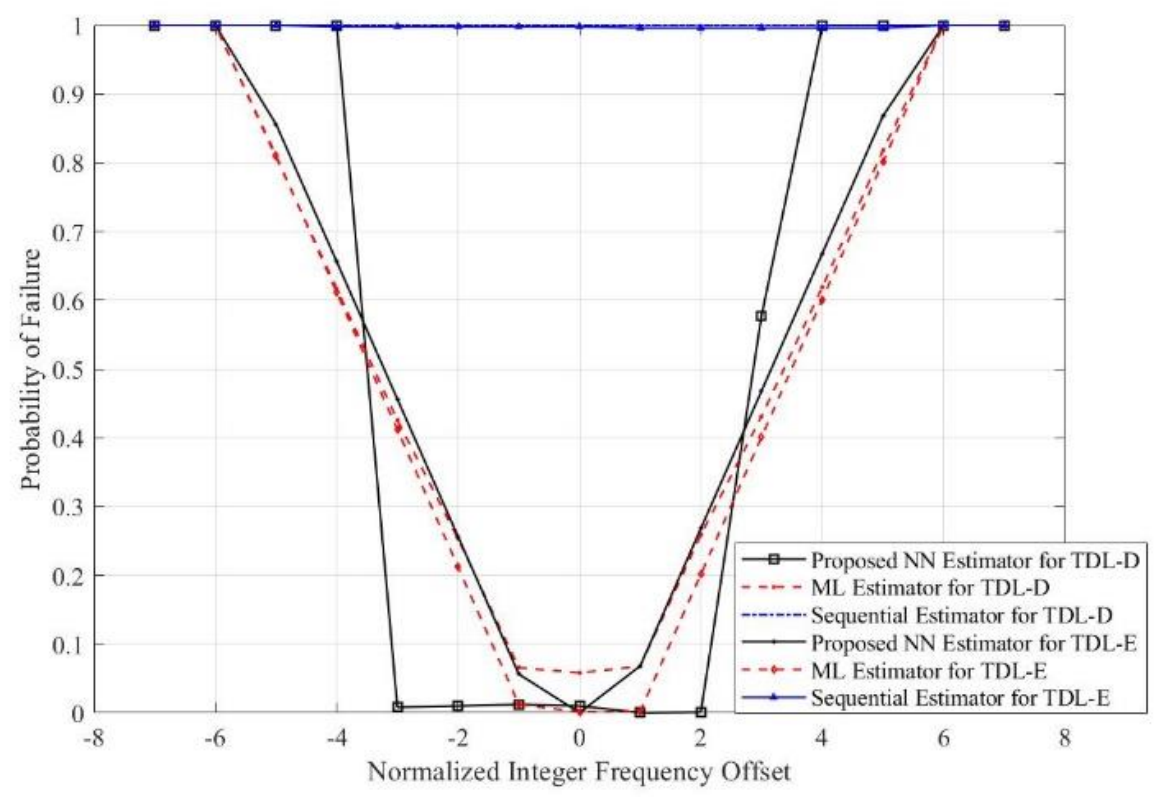

Figure. $10 \mathrm{P}_{\mathrm{f}}$ vs. Normalized IFO for LOS delay profiles

delay spreads. From simulation results, it is affirmatively proved that the proposed $\mathrm{NN}$ estimator outperforms in all channel models. With an increase in the deep learning approach for the physical layer of $5 \mathrm{G} \mathrm{NR}$, this proposed method for estimation of IFO and detection of PSS could be employed to reduce synchronization errors.

\section{Conflicts of interest}

The authors declare no conflict of interest

\section{Author contributions}

Vibha Patel, the first author contributed in conceptualization, methodology, software, validation, formal analysis, investigations, writing-original draft preparation, writing-review and editing and visualization.

Krishna Warhade, the second author supervised and administered project. 


\section{References}

[1] 3GPP TS 38.211, NR; Physical Channels and Modulation (Release 15), Version.15.4.0, pp. 1$97,2019$.

[2] 3GPP TS 38.212, NR; Multiplexing Channel Coding (Release 15), Version.15.4.0, pp. 1-102, 2019

[3] 3GPP TS 38.213, NR; Physical Layer Procedures for Control (Release 15), Version.15.4.0, pp. 1-105, 2019

[4] 3GPP TS 38.104, NR; Base Station (BS) radio transmission and reception (Release 15), Version 15.2.0, pp. 1-155, 2018

[5] H. Abdzadeh-Ziabari, W. Zhu and M. N. S. Swamy, "Joint Maximum Likelihood Timing, Frequency Offset, and Doubly Selective Channel Estimation for OFDM Systems", IEEE Transactions on Vehicular Technology, Vol. 67, No. 3, pp. 2787-2791, 2018.

[6] D. Li, Y. Li, H. Zhang, L. J. Cimini, and Y. Fang, "Integer Frequency Offset Estimation for OFDM Systems with Residual Timing Offset Over Frequency Selective Fading Channels", IEEE Transactions on Vehicular Technology, Vol. 61, No. 6, pp. 2848-2853, 2012.

[7] L. Cheng, B. Henty, F. Bai, and D. D. Stancil, "Doppler spread and coherence time of rural and highway vehicle-to-vehicle channels at 5.9 GHz", In: Proc. of 2013 IEEE Global Telecommunications Conference, LA, USA, pp. 1-6, 2008.

[8] P. K. Nishad and P. Singh, "Carrier frequency offset estimation in OFDM systems", In: Proc. of 2013 IEEE Conference on Information \& Communication Technologies, Thuckalay, India, pp. 885-889, 2013.

[9] S. Tanangsanakool, P. Reangsuntea, K Mori, and P. Boonsrimuang, "Low-Complexity based TDE Method for OFDM Signal in Higher TimeVarying Fading Channels", International Journal of Intelligent Engineering \& Systems, Vol. 13, No.4, pp. 21-32, 2020.

[10] A. Omri, M. Shaqfeh, A. Ali, and H. Alnuweiri, "Synchronization Procedure in 5G NR Systems", in IEEE Access, Vol. 7, pp. 41286-41295, 2019.

[11] T. Wang, C. K. Wen, H. Wang, F. Gao, T. Jiang, and S. Jin, "Deep learning for wireless physical layer: Opportunities and challenges", China Commun., Vol. 14, No. 11, pp. 92-111, 2017.

[12] H. Huang, S. Guo, G. Gui, Z. Yang, J. Zhang, H. Sari, and F. Adachi, "Deep Learning for
Physical-Layer 5G Wireless Techniques: Opportunities, Challenges and Solutions", IEEE Wireless Communications, Vol. 27, No.1, pp. 214-222, 2020.

[13] M. Morelli and M. Moretti, “A Robust Maximum Likelihood Scheme for PSS Detection and Integer Frequency Offset Recovery in LTE Systems", IEEE Transactions on Wireless Communications, Vol. 15, No. 2, pp. 1353-1363, 2016.

[14] C. Chu, I. Lai, Y. Lan, and T. Chiueh, "Efficient Sequential Integer CFO and Sector Identity Detection for LTE Cell Search", IEEE Wireless Communications Letters, Vol. 3, No. 4, pp. 389392, 2014.

[15] Y. A. Jung, D. Shin, and Y. H. You, "A Computationally Efficient Joint Cell Search and Frequency Synchronization Scheme for LTE Machine-Type Communications", Symmetry, Vol. 11, No. 11, p. 1394, 2019.

[16] Y. H. You and H. K. Song, "Efficient Sequential Detection of Carrier Frequency Offset and Primary Synchronization Signal for 5G NR Systems", in IEEE Transactions on Vehicular Technology, Vol. 69, No. 8, pp. 9212-9216, 2020.

[17] R. M. Dreifuerst, R. W. Heath, M. N. Kulkarni, and J. Charlie, "Deep Learning-based Carrier Frequency Offset Estimation with One-Bit ADCs", In: Proc. of 2020 IEEE 21st International Workshop on Signal Processing Advances in Wireless Communications (SPAWC), GA, USA, pp. 1-5, 2020.

[18] V. Ninkovic, D. Vukobratovic, A. Valka, and D. Dumic, "Deep learning based packet detection and carrier frequency offset estimation in IEEE 802.11ah", arXiv, identifier arXiv: 2004. 11716v1, 2020.

[19] J. Liu, K. Mei, X. Zhang, D. McLernon, D. Ma, J. Wei, and S. A. R. Zaidi, "Fine timing and frequency synchronization for MIMO-OFDM: An extreme learning approach", submitted to IEEE Transactions on Wireless Communications, identifier arXiv: 2007. 09248.

[20] M. Zhou, X. Huang, Z. Feng, and Y. Liu, "Coarse Frequency Offset Estimation in MIMO Systems Using Neural Networks: A Solution with Higher Compatibility", in IEEE Access, Vol. 7, pp. 121565-121573, 2019.

[21] J. J. V D Beek, M. Sandell, and P. O. Borjesson, "ML estimation of time and frequency offset in 
Received: May 25, 2021. Revised: July 12, 2021.

OFDM systems", in IEEE Transactions on Signal Processing, Vol. 45, No. 7, pp. 1800$1805,1997$.

[22] 3GPP TR 38.900, 5G; Study on channel model for frequency spectrum above $6 \mathrm{GHz}$ (Release 14), Version 14.2.0, pp. 1-88, 2017 